\title{
Error Control for a Designed State Estimator Function Block of Foundation Fieldbus
}

\author{
M. Mahmoud, N. El-Amary, H. Issa \\ Electrical And Control Engineering, AASTMT, Cairo, Egypt
}

\begin{abstract}
Design and simulation of a Foundation Fieldbus State Estimator Function Block (FFSEFB), which can control and minimize the system estimated states' error is proposed in this paper. Due to the importance of the foundation fieldbus systems in manufacturing field, there is a great need for extending their uploaded signals data capacity with high accuracy. A state estimator function block is designed, simulated and analyzed using Matlab/Simulink program to be adapted with a foundation fieldbus of any dynamic system. The designed block contains an adapted PI controller to eliminate the estimated error and ensures a smooth stable block response. The adapted controller block is added in different locations to eliminate the error results from the estate estimator. The controller parameters are changed according to the system input signal to ensure over-damped estimated output signal with zero error. Artificial Neural Network (ANN) technique is utilized in controller parameters determination. The developed intelligent controlled state estimator function block is tested on a $3^{\text {rd }}$ order dynamic system, and the results presents satisfying output.
\end{abstract}

The block design and optimization using Matlab/Simulink and is implemented to an impeded system.

Key word-Foundation Fieldbus (FF), State Estimator, Function Block (SEFB), adaptive PID controller, feedback system, Artificial Neural Network (ANN) technique

\section{INTRODUCTION}

In 1980s, the development of Foundation Fieldbus (FF) was started by several DCS vendors based on the complete digital communication concept [1]. In 1992 an international group, the Interoperable Systems Project (ISP) was founded to create an internationally uniform fieldbus standard. At the same time, the manufacturers and users of the French Flux Information Processes (FIP) established the international user organization WorldFIP. In 1994, for technical, economic and political reasons, the ISP and the WorldFIP were merged to form the Fieldbus Foundation (FF) [2]. The FF is an independent notfor-profit organization that defines $\mathrm{FF}$ specifications and certifies products compliant with the standard [2]. FF is a full digital two-way, multi -drop communication system for plant operation equipment. $\mathrm{FF}$ is used in continuous processes control like level, temperature and flow control. These types of process are found in oil \& Gas, chemical and food plants. FF can also be used for monitoring over long distances [3].

FF has two communication protocols: The first, H1, transmits at $31.25 \mathrm{~Kb} / \mathrm{s}$ and is used to connect the field devices.
The second, High Speed Ethernet (HSE), uses 10 or $100 \mathrm{Mbps}$ Ethernet as the physical layer and provides a high-speed backbone for the network [3].

A variety of fieldbus technologies and digital fieldbus devices have been introduced within the industrial process systems over the last 10 years [4]. There has been a gradually acceptance of the fact that a variety of communication technologies are needed to fully address the applications' requirements of a manufacturing facility. FF systems which are applied to continuous real time processes are used in the majority of applications which use a group of function blocks that execute at a specified rate within a Fieldbus device or distributed across the Fieldbus network like PI and PID, as well as compensators [5] [6].

State estimation (SE) is the process of determining unknown values of a system state variable based on previous measurements [7]. SE plays an important role in the monitoring and control of industrial process systems in order to increase the availability.

The approach of dynamic process control by state variables is the basis of modern control theory, which started in the 50's. It started in aerospace researches aiming at the development of more specialized and more accurate control systems. The use of this type of controllers is limited to digital environments, and consequently appropriate for foundation fieldbus.

Aiming at the expansion of possibilities to implement foundation fieldbus and the resources of this system, this paper introduces the design, optimization and implementation of a modern dynamic compensator characteristic of control systems by state variables. Furthermore, the state estimator function block is described with mathematical equations. Adaptive PI controller block is added in different locations to eliminate the error results smoothly from the estate estimator.

\section{STATE ESTIMATOR AND FEEDBACK SYSTEM}

Design of state estimator block is processed according to the requirements quoted in the Foundation Fieldbus (FF) standards and modern control theory fundamentals.

The dynamic system is described using state space representation as follows [8]: 


$$
\begin{aligned}
& x(s)=A \cdot x(s)+B \cdot u(s) \\
& y(s)=C \cdot x(s)+D \cdot u(s)
\end{aligned}
$$

Where;

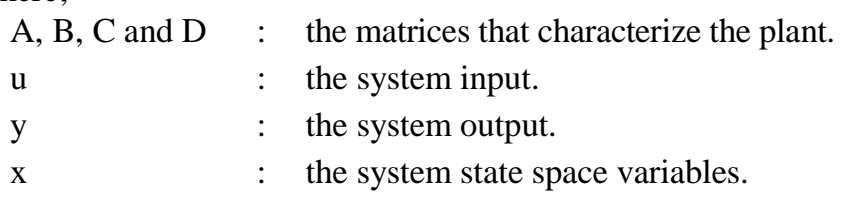

For feedback regulating system implementation, the operating input of the system is defined using the feedback matrix $\mathrm{K}$ as follows:

$$
u(s)=r(s)+K . x(s)
$$

A state estimator is used, where it is not possible to measure the states, and thus the state variables will be estimated $\left(x_{e s t}\right)$ and equation (3) will be as follows:

$$
u(s)=r(s)+K \cdot x_{e s t}(s)
$$

Applying equation (4), equation (1) and (2) will be as follows:

$$
\begin{gathered}
\dot{x}(s)=A \cdot x(s)+[B \cdot K] x_{e s t}(s)+[B] \cdot r(s) \\
y(s)=C \cdot x(s)+D \cdot r(s)+[D \cdot K] x_{e s t}(s)
\end{gathered}
$$

The used state estimator shows the dynamic system behavior as described in the following equation.

$$
\begin{gathered}
x_{e s t}(s)=A \cdot x_{e s t}(s)+B \cdot u(s)-L \cdot\left(y(s)-y_{e s t}(s)\right) \\
y_{e s t}(s)=C \cdot x_{e s t}(s)
\end{gathered}
$$

Where;

$$
\text { L : the feedback estimator matrix. }
$$

$$
y(s)-y_{e s t}(s)=C \cdot x(s)+D \cdot r(s)+[D \cdot K] x_{e s t}(s)-C \cdot x_{e s t}(s)
$$

$\dot{x}_{e s t}(s)=A \cdot x_{e s t}(s)+[B]\left[r(s)+[K] . x_{e s t}(s)\right]-[L . C] x(s)-[L . D] r(s)$ $-[$ L.D.K $] \cdot x_{\text {est }}(s)+[$ L.C $] . x_{\text {est }}(s)$

$$
\dot{x}_{\text {est }}(s)=-[L C] x(s)+[A+B K-L D K+L C] x_{e s t}(s)+[B-L D] r(s)
$$

The complete dynamic system formed by the plant with the feedback states and a parallel estimator function block can be described by the following matrix.

$$
\left[\begin{array}{c}
\dot{x} \\
x_{\text {est }}
\end{array}\right]=\left[\begin{array}{cc}
A & B K \\
-L C & A+B K-L D K+L C
\end{array}\right] \cdot\left[\begin{array}{c}
x \\
x_{\text {est }}
\end{array}\right]+\left[\begin{array}{c}
B \\
B-L D
\end{array}\right] \cdot r
$$

Figure 1 shows an illustration of the Matlab/Simulink block diagram of the state estimator function block and Fig. 5 shows the error signal.

\section{STATE ESTIMATOR ERROR CONTROL}

The target of this paper is to detect the complete pattern of the output signal through estimating the missed samples with high accuracy. An adaptive Proportional, Integral and Derivative (PID) controller is utilized to eliminate the error [5], which is generated from the differences between the actual supposed output (y) of the plant and the estimated output of the state estimator (yest). Three different locations are proposed for the PID controller. It is observed that there is no need for the Derivative (D) controller in the three locations. The three locations are;

A- the input of the state estimator

B- the output of the estate estimator

C- the feedback loop of the difference between the plant output and the state estimator output.

\section{A. Adding PI Controller to the input signal of the estimator}

The Matlab/Simulink block diagram of the system with the PI controller installed at the input entrance of the state estimator is shown in Fig. 2.

\section{From Fig. 2;}

$$
\left(x_{\text {est }}(s)\right)_{\text {con }}=\left[K_{P}+\frac{K_{I}}{s}\right] x_{e s t}(s)
$$

The error which is the difference between the actual system output and the output of the estimator model is equal to;

$$
\begin{aligned}
& E(s)=y(s)-\left[y_{e s t}(s)\right]_{c o n} \\
& E(s)=C \cdot x(s)+\left[D \cdot K \cdot K_{P}-C \cdot K_{P}\right] \cdot x_{e s t}(s) \\
& +\left[D . K . K_{I}-C \cdot K_{I}\right] \frac{x_{e s t}(s)}{s}+D \cdot r(s)
\end{aligned}
$$

To eliminate the steady state error of the estimated signal;

$$
\operatorname{Lim}_{s \rightarrow 0} s . E(s)=0
$$

$$
\begin{aligned}
& \underset{s \rightarrow 0}{\operatorname{Lim}} s \cdot E(s)= \\
& \text { C. } \operatorname{Lim}_{s \rightarrow 0} s \cdot x(s)+\left[D \cdot K \cdot K_{P}-C \cdot K_{P}\right] \cdot \underset{s \rightarrow 0}{\operatorname{Lim}} s \cdot x_{e s t}(s) \\
& +\left[D \cdot K . K_{I}-C \cdot K_{I}\right] \operatorname{Lim}_{s \rightarrow 0} x_{e s t}(s)+D \cdot \underset{s \rightarrow 0}{\operatorname{Lim}} \operatorname{s.r}(s)=0
\end{aligned}
$$


From equation (17); $x_{\text {est }}(s)$ should be impulse signal otherwise the equation will tends to infinity.

$$
\text { C. } \operatorname{Lim}_{s \rightarrow 0} s \cdot x(s)+[D \cdot K-C]\left[K_{I}\right] \cdot \operatorname{Lim}_{s \rightarrow 0} x_{e s t}(s)+D \cdot \operatorname{Lim}_{s \rightarrow 0} s \cdot r(s)=0
$$

Where;

F is the amplitude of the $x_{e s t}(s)$

$K_{I}=-\frac{1}{F}[D \cdot K-C]^{-1} \cdot\left[C \cdot \operatorname{Lim}_{s \rightarrow 0} s \cdot x(s)+D \cdot \operatorname{Lim}_{s \rightarrow 0} \operatorname{s.r}(s)\right]$

From the above equation; $K_{I}$ value will be one of the following cases:

1. $\quad x$ and $r$ are impulse signals:

$$
K_{I}=0 \text {. }
$$

2. $x$ and $r$ are step signals:

$$
K_{I}=-\frac{1}{F}[D \cdot K-C]^{-1} \cdot[C \cdot G+D \cdot H]
$$

Where;

$\mathrm{G}$ and $\mathrm{H}$ are the amplitudes of $x$ and $r$ respectively.

3. $\quad x$ is a step signal while $r$ is an impulse signals:

$$
K_{I}=-\frac{G}{F}[D \cdot K-C]^{-1} \cdot[C]
$$

4. $\quad x$ is an impulse signal while $r$ is a step signals:

$$
K_{I}=-\frac{H}{F}[D \cdot K-C]^{-1} \cdot[D]
$$

$$
\text { Otherwise } K_{I}=\infty
$$

$x_{e s t}(s)=A \cdot x_{e s t}(s)+B \cdot u(s)-L\left(y(s)-y_{e s t}(s)\right)$

During signal estimation, $y(s)=0$

$\dot{x}_{e s t}(s)=\left[s . I-A . K_{P}-\frac{A . K_{I}}{s}-L . C . K_{P}-\frac{L . C \cdot K_{I}}{s}\right]^{-1} \cdot[B]$

$\frac{\left(y_{\text {est }}(s)\right)_{c o n}}{u(s)}=s .[C] \cdot\left[s^{2} \cdot I-\left(A \cdot K_{P}+L \cdot C \cdot K_{P}\right) \cdot s-\left(A \cdot K_{I}+L \cdot C \cdot K_{I}\right)\right]^{-1} \cdot[B]$

The closed loop characteristic equation of the simulated system is:

$$
s^{2} . I-\left[A . K_{P}+\text { L.C.K } K_{P}\right] s-\left[A . K_{I}+\text { L.C. } K_{I}\right]=0
$$

For the stability of the system, poles should be positive

$$
K_{P} \succ \sqrt{\frac{-4 . K_{I}}{[A+L . C]}}
$$

By considering the values of $K_{I}, K_{P}$ will be one of the following;

1. When $K_{I}=0$

$$
K_{P} \succ 0
$$

2.

When $K_{I}=-\frac{1}{F}[D \cdot K-C]^{-1} \cdot[C \cdot G+D \cdot H]$

$$
K_{P} \succ \sqrt{\frac{\frac{4}{F}[D \cdot K-C]^{-1} \cdot[C . G+D \cdot H]}{[A+L . C]}}
$$

3.

$$
\text { When } K_{I}=-\frac{G}{F}[D \cdot K-C]^{-1} \cdot[C]
$$

$$
K_{P} \succ \sqrt{\frac{\frac{4 G}{F} \cdot[D \cdot K-C]^{-1} \cdot[C]}{[A+L . C]}}
$$

4.

$$
\text { When } K_{I}=-\frac{H}{F}[D \cdot K-C]^{-1} \cdot[D]
$$

$$
K_{P} \succ \sqrt{\frac{\frac{4 H}{F} \cdot[D \cdot K-C]^{-1} \cdot[D]}{[A+L . C]}}
$$

\section{B. Adding PI Controller to the error signal of the estimator}

Figure 3 illustrates the Matlab/Simulink block diagram of the system with the PI controller installed through the error signal of the state estimator. As shown in Fig. 3:

$$
\left[y(s)-y_{\text {est }}(s)\right]_{c o n}=\left[y(s)-y_{\text {est }}(s)\right] . P I
$$




$$
\begin{aligned}
& {\left[y(s)-y_{e s t}(s)\right]_{c o n}=\left[C \cdot K_{P}+C \cdot \frac{K_{I}}{s}\right] \cdot x(s)} \\
& +\left[D \cdot K \cdot K_{P}-C \cdot K_{P}+\frac{D \cdot K \cdot K_{I}}{s}-\frac{C \cdot K_{I}}{s}\right] \cdot x_{e s t}(s) \\
& +\left[D \cdot K_{P}+\frac{D \cdot K_{I}}{s}\right] \cdot r(s) \\
& E(s)=\left[y(s)-y_{e s t}(s)\right]_{c o n} \\
& E(s)=\left[C \cdot K_{P}+C \cdot \frac{K_{I}}{s}\right] \cdot x(s) \\
& +\left[D \cdot K \cdot K_{P}-C \cdot K_{P}+\frac{D \cdot K \cdot K_{I}}{s}-\frac{C \cdot K_{I}}{s}\right] \cdot x_{e s t}(s) \\
& +\left[D \cdot K_{P}+\frac{D \cdot K_{I}}{s}\right] \cdot r(s) \\
& \operatorname{Lim}_{s \rightarrow 0} s . E(s)=\left[C \cdot K_{P}+C \cdot \frac{K_{I}}{s}\right] \cdot \operatorname{Lim}_{s \rightarrow 0} s \cdot x(s)+ \\
& {\left[D . K . K_{P}-C \cdot K_{P}+\frac{D \cdot K \cdot K_{I}-C \cdot K_{I}}{s}\right] . \operatorname{Lim}_{s \rightarrow 0} s . x_{e s t}(s)} \\
& +\left[D \cdot K_{P}+\frac{D \cdot K_{I}}{S}\right] \cdot \operatorname{Lim}_{s \rightarrow 0} \operatorname{s.r}(s)=0
\end{aligned}
$$

From (29); $x_{\text {est }}(s), x(s)$, and $r(s)$ should be impulse signal otherwise the equation will tend to infinity.

Therefore;

$$
\begin{gathered}
{\left[C \cdot K_{I}\right] \cdot G+\left[D \cdot K \cdot K_{I}-C \cdot K_{I}\right] \cdot F+\left[D \cdot K_{I}\right] H=0} \\
K_{I}=0
\end{gathered}
$$

The new dynamic system behavior equation will be described as in the following equation

$$
\begin{gathered}
\dot{x}_{e s t}=A \cdot x_{e s t}+B . u-L\left(y-y_{e s t}\right)_{c o n} \\
\dot{x}_{e s t}=[A] x_{e s t}(s)+[B] \cdot u(s)-\left[L . C . K_{P}\right] \cdot x(s) \\
-\left[\text { L.D.K.K } K_{P}-\text { L.C.K. }{ }_{P}\right] \cdot x_{e s t}(s)-\left[L . D . K_{P}\right] \cdot r(s)
\end{gathered}
$$

$$
\begin{aligned}
& {\left[s . I-A-L . D . K . K_{P}-L . C . K_{P}\right] \cdot x_{e s t}(s)} \\
& =[B] . u(s)-\left[L . D . K_{P}\right] \cdot r(s)-\left[L . C . K_{P}\right] x(s)
\end{aligned}
$$

When $\mathrm{x}(\mathrm{s})=0$

$$
\begin{aligned}
& {\left[s . I-A-L \cdot D \cdot K \cdot K_{P}-\text { L.C.K } K_{P}\right] x_{e s t}(s)} \\
& =[B] \cdot u(s)-\left[\text { L.D. } K_{P}\right] \cdot r(s)
\end{aligned}
$$

As

$$
\begin{gathered}
y_{e s t}=C \cdot x_{e s t} \\
{\left[s . I-A-L \cdot D \cdot K \cdot K_{P}-L \cdot C \cdot K_{P}\right] \cdot[C] \cdot y_{e s t}(s)} \\
=[B] \cdot u(s)-\left[L \cdot D \cdot K_{P}\right] \cdot r(s) \\
\frac{y_{e s t}(s)}{u(s)}=\left[[B]-\left[L . C . K_{P}\right] \cdot \frac{r(s)}{u(s)}\right]\left[\left[s \cdot I-A-L \cdot D \cdot K \cdot K_{P}-L \cdot C \cdot K_{P}\right]\left[[C]^{-1}\right]^{-1}\right.
\end{gathered}
$$

The system characteristic equation is

$$
\left[A+L . D . K . K_{P}+\text { L.C.K } K_{P}\right]=0
$$

For the stability of the system, poles should be positive

$$
\left[K_{P}\right] \succ \frac{[-A]}{[L . C+\text { L.D.K }]}
$$

As long as the value of $K_{I}=0$, the signal is unstable. This location for the controller is not preferred.

The error signal of the Matlab/Simulink block diagram is shown in Fig. 7. From the figure it can be noticed that short burst of oscillations (transient signal) occurs in the begging before the system becomes unstable.

\section{Adding PI Controller to the output signal of the estimator}

The Matlab/Simulink block diagram of the system with the PI controller installed at the output signal of the state estimator is shown in Fig. 4.

$$
\begin{gathered}
\left(y_{\text {est }}\right)_{\text {con }}=[C]\left[K_{P}+\frac{K_{I}}{s}\right] \cdot x_{e s t}(s) \\
\text { Error }=y(s)-\left(y_{\text {est }}\right)_{c o n} \\
E(s)=[C] x(s)+\left[D \cdot K-C K_{P}\right] \cdot x_{\text {est }}(s)-\left[C \cdot K_{I}\right] \frac{x_{\text {est }}(s)}{s}+[D] \cdot r(s)
\end{gathered}
$$


$\operatorname{Lim}_{S \rightarrow 0} s \cdot E(s)=C \cdot \operatorname{Lim}_{S \rightarrow 0} s \cdot x(s)+\left[D \cdot K-C K_{P}\right] \operatorname{Lim}_{S \rightarrow 0} s \cdot x_{e s t}(s)$

$-\left[C . K_{I}\right] \operatorname{Lim}_{S \rightarrow 0} x_{e s t}(s)+D . \underset{S \rightarrow 0}{\operatorname{Lim}} s . r(s)=0$

As shown in (42), $x_{\text {est }}(s)$ should be impulse signal otherwise the equation will tend to infinity

C. $\operatorname{Lim}_{s \rightarrow 0} s \cdot x(s)-\left[C \cdot K_{I}\right] \operatorname{Lim}_{s \rightarrow 0} x_{e s t}(s)+D \cdot \operatorname{Lim}_{s \rightarrow 0} s \cdot r(s)=0$

$\left[C . K_{I}\right] \operatorname{Lim}_{S \rightarrow 0} x_{e s t}(s)=C \cdot \operatorname{Lim}_{S \rightarrow 0} s . x(s)+D \cdot \operatorname{Lim}_{S \rightarrow 0} s . r(s)=0$

$K_{I}=\frac{1}{F}\left[\operatorname{Lim}_{S \rightarrow 0} s \cdot x(s)+[C]^{-1}[D] \operatorname{Lim}_{S \rightarrow 0} \operatorname{s.r}(s)\right]$

Where; $\mathrm{F}$ is the amplitude of the $x_{\text {est }}(s)$.

From (45), $K_{I}$ value will be one of the following cases:

1. $\quad x$ and $r$ are impulse signals:

$$
K_{I}=0 \text {. }
$$

2. $\quad x$ and $r$ are step signals:

$$
K_{I}=\frac{1}{F}\left[[I] \cdot G+[C]^{-1} \cdot[D] H\right]
$$

Where;

$\mathrm{G}$ and $\mathrm{H}$ are the amplitudes of $x$ and $r$ respectively.

3. $\quad x$ is a step signal while $r$ is an impulse signals:

$$
K_{I}=\frac{G}{F} \cdot[I]
$$

4. $\quad x$ is an impulse signal while $r$ is a step signals:

$$
K_{I}=\frac{H}{F} \cdot[C]^{-1} \cdot[D]
$$

Otherwise $K_{I}=\infty$

$$
\dot{x}_{e s t}(s)=B . u(s)-[L] \cdot\left[y(s)-y_{e s t}(s)\right]+A \cdot x_{e s t}(s)
$$

During signal estimation, $y(s)=0$

$$
x_{\text {est }}(s)=\left[I . s-A-L . C . K_{P}-\frac{\text { L.C. } K_{I}}{s}\right]^{-1} \cdot[B] \cdot u(s)
$$

$$
\frac{\left(y_{e s s}\right)_{c o n}}{u(s)}=[C]\left[K_{P}+\frac{K_{I}}{s}\right]\left[\left[I . s-A-L . C \cdot K_{P}-\frac{L \cdot C \cdot K_{I}}{s}\right]^{-1} \cdot[B]\right.
$$

The closed loop characteristic equation of the simulated system is:

$$
\begin{aligned}
& {[I] s^{2}-\left[A+L . C . K_{P}\right] \cdot s-\left[L . C . K_{I}\right]=0} \\
& s_{1,2}=\frac{\left[A+L \cdot C \cdot K_{P}\right] \pm \sqrt{\left[A+L \cdot C \cdot K_{P}\right]^{2}+4 \cdot\left[L \cdot C \cdot K_{I}\right]}}{2}
\end{aligned}
$$

1. When $K_{I}=0$.

$$
\left[K_{P}\right] \frac{-[A]}{[L] \cdot[C]}
$$

2. When $K_{I}=\frac{1}{F} \cdot\left[[I] \cdot G+[C]^{-1} \cdot[D] \cdot H\right]$

$$
\begin{aligned}
& {\left[A+L . C . K_{P}\right]+\sqrt{\left[A+L . C . K_{P}\right]^{2}+4 \cdot \frac{G}{F}[L] \cdot[C]+4 . H .[L] \cdot[D]}<0} \\
& {\left[A+L . C . K_{P}\right]-\sqrt{\left[A+L . C . K_{P}\right]^{2}+4 \cdot \frac{G}{F}[L][C]+4 . H .[L][[D]}<0}
\end{aligned}
$$

3. When $K_{I}=\frac{G}{F} \cdot[I]$.

$$
\begin{aligned}
& {\left[A+L . C . K_{P}\right]+\sqrt{\left[A+L . C . K_{P}\right]^{2}+4 \cdot[L] \cdot[C] \cdot \frac{G}{F}<0}} \\
& {\left[A+L . C . K_{P}\right]-\sqrt{\left[A+L . C . K_{P}\right]^{2}+4 \cdot[L][C] \cdot \frac{G}{F}<0}}
\end{aligned}
$$

4.

When $K_{I}=\frac{H}{F} \cdot[C]^{-1} \cdot[D]$

$$
\begin{aligned}
& {\left[A+L . C . K_{P}\right]+\sqrt{\left[A+L . C . K_{P}\right]^{2}+4 \cdot[L] \cdot \frac{H}{F} \cdot[D]<0}} \\
& {\left[A+L . C . K_{P}\right]-\sqrt{\left[A+L . C . K_{P}\right]^{2}+4 \cdot[L] \cdot \frac{H}{F} \cdot[D] 0}}
\end{aligned}
$$

\section{SIMULATION AND RESULTS}

The three studied controlled system models are simulated using Matlab/Simulink. Different values of PI controller gains $\left(\mathrm{K}_{\mathrm{P}}\right.$ and $\left.\mathrm{K}_{\mathrm{I}}\right)$ are tested for the three controller locations. The effect of the different controller parameters values through the three different locations on the estimated error signal is shown in Fig. 6, 7 and 8. 
The error signal of the Matlab/Simulink block diagram where the PI controller is installed at the input signal of the state estimator is shown in Fig. 6. From the fugre it can be noticed that short burst of oscillations (transient signal) occurs in the begging before the system becomes stable. Steady state error is constant.

The error signal of the Matlab/Simulink block diagram where the PI controller is installed through the error signal is shown in Fig. 7. From the figure. it can be noticed that short burst of oscillations (transient signal) occurs in the begging before the system becomes unstable.

The error signal of the Matlab/Simulink block diagram where the PI controller is installed at the output signal of the state estimator is shown in Fig. 8. From the figure it can be noticed that the system is unstable and an error is always exist, Steady state error is not constant.

The results shows that the best location for the PI controller block is at the input signal of the estimator. Values of the P and I can be calculated as detailed above.

\section{CONCLUSION}

A newly developed adapted PI controlled State Estimator Function Block (SEFB) for Foundation Fieldbus is designed and simulated in this paper.

As the integration of tools from modern control theory with Foundation Fieldbus protocol has shown to be viable, due to its great benefits, the newly developed system is completely derived and simulated utilizing the modern control concepts. An adaptable bi-variables PI controller is applied to three different locations of the simulated foundation fieldbus system to extend the uploaded signals data capacity of the system and improve the estimated signal of the state estimator function block. The developed system with the three tested locations of the adapted PI controller is derived and then simulated utilizing Matlab/Simulink program. The tested three locations of the PI controller are; i- the input of the state estimator, ii- the output of the estate estimator, and iii- the feedback loop of the difference between the plant output and the state estimator output.

The main target of the adaptable PI controller (in each location) is to eliminate the error of the estimated output signal and ensure a smooth over damped stable block response. The derived models of the controller parameters (in each case) show very promising results when they are tested on the Matlab/Simulink models.

The developed intelligent controlled state estimator function block is applied and tested on a $3^{\text {rd }}$ order dynamic system. The results illustrate acceptable output.

\section{REFERENCES}

[1] Yoshinori Sato, "Engineering Experience in Foundation Fieldbus Technology", ICROS-SICE International Joint Conference, Fukuoka International Congress Center, Japan, 2009, PP 2087 - 2090.

[2] FOUNDATION Fieldbus Technical Overview, Revision 3.0, 2003.

[3] FOUNDATION Fieldbus, 4th ed., Samson, Frankfurt, Germany, 2000.

[4] Foundation Fieldbus, 1st ed., YOKOGAWA, Manama, Bahrain, 2004.

[5] Amphawan Julsereewong, Prasit Julsereewong, and Thipat Dejsiriphant, "Study on Field Control Locations for Foundation Fieldbus-Based Cascade Control Loop", SICE Annual Conference, Akita University, Akita, Japan, 2012, PP 1706 1711.

[6] Suvishchan Arayawat, Teerawat Thepmanee, Prasit Julsereewong, and Amphawan Julsereewong, "Process Alarming in PID Loop Using Foundation Fieldbus with Control in the Field: A Case Study of DeltaV Host System", Proceedings of the IEEE/SICE International Symposium on System Integration, Chuo University, Tokyo, Japan, 2014, PP 749 754.

[7] Foundation Fieldbus Overview, National Instruments, Austin, Texas, 2003, pp. 370729A-01

[8] Mário Pinotti Jr. and Dennis Brandão. "State Estimator Function Block on Control Loops Fieldbus Foundation Protocol", Proceedings of the First ISA/IEEE Conference for Sensors for Industry, Rosemont, IL, 2001, PP 134 - 136.

[9] Function Block Capabilities in Hybrid/Batch Applications, 1.1 ed., 4 December 2002

[10] Foundation Specification - Function Block Message Specification - FF-870-1.1

[11] Foundation Specification - Function Block Application Process Part 1 - FF-890-1.2

[12] Foundation Specification - Function Block Application Process Part 2 - FF-891-1.2

[13] Foundation Specification - Function Block Application Process Part 3

[14] Foundation Specification Function Block Application Process Part 4. 


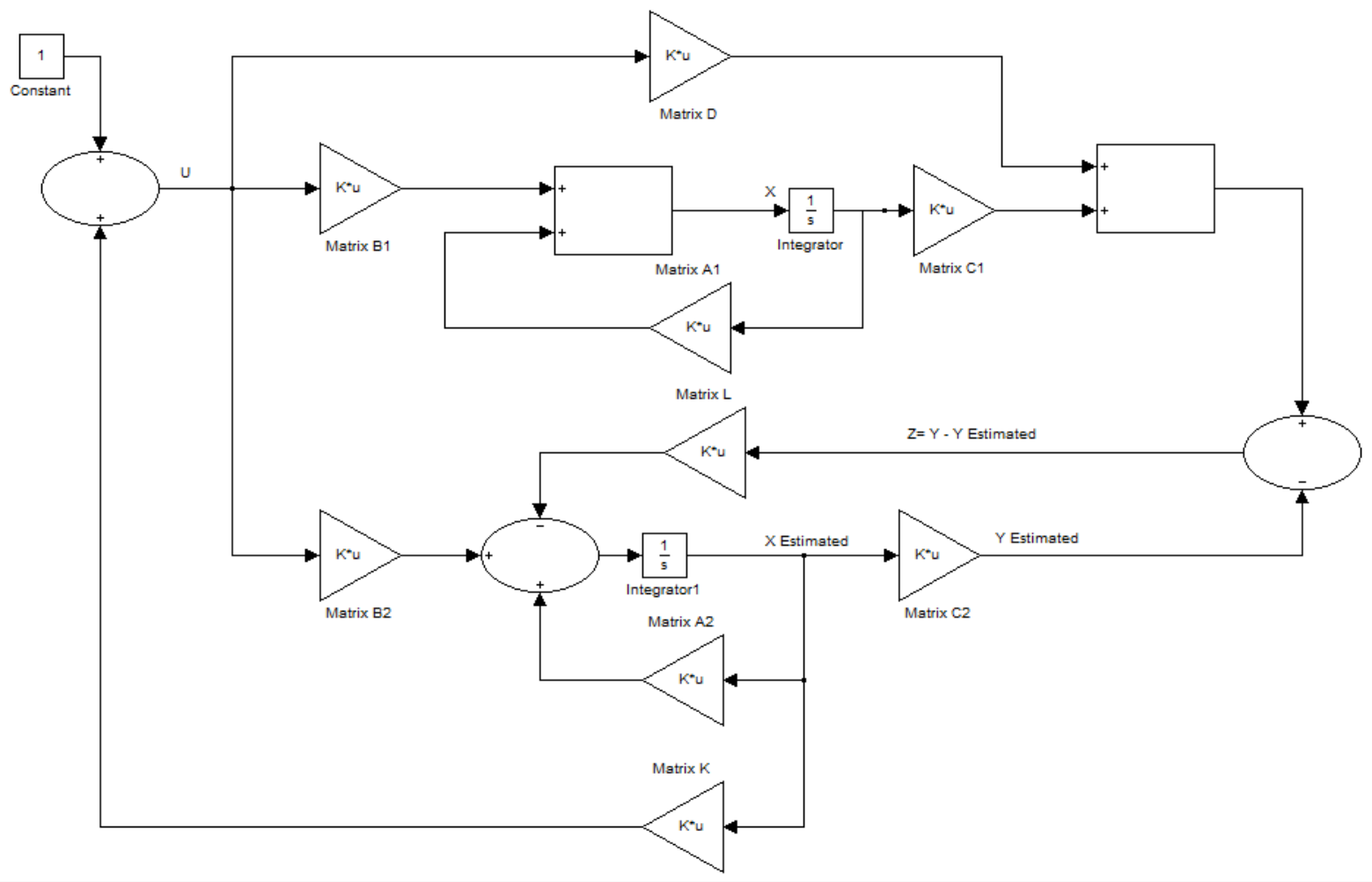

Fig. 1. State Estimator Function Block

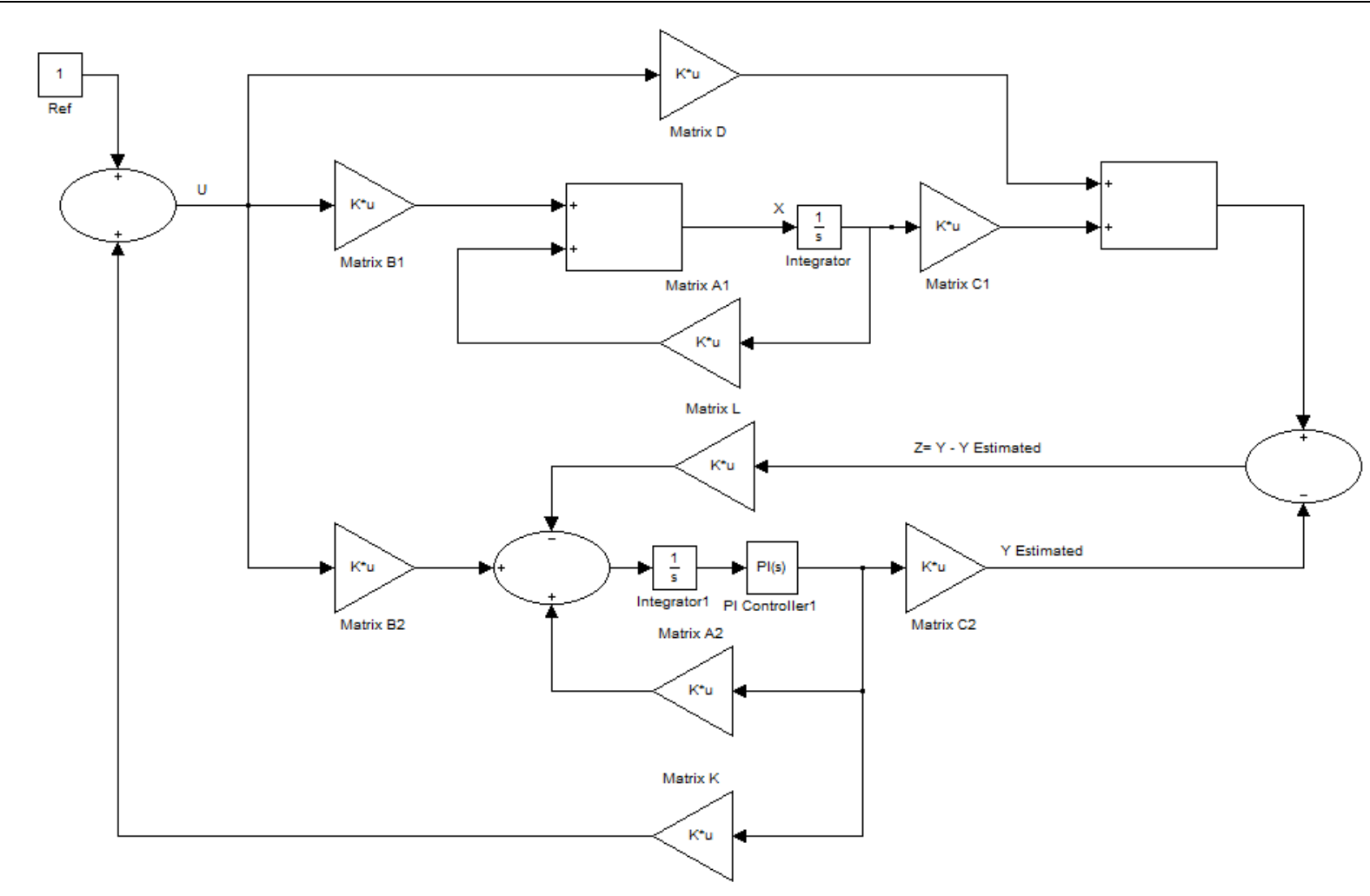

Fig. 2. State estimator function block with PI controller at the input signal of the state estimator 


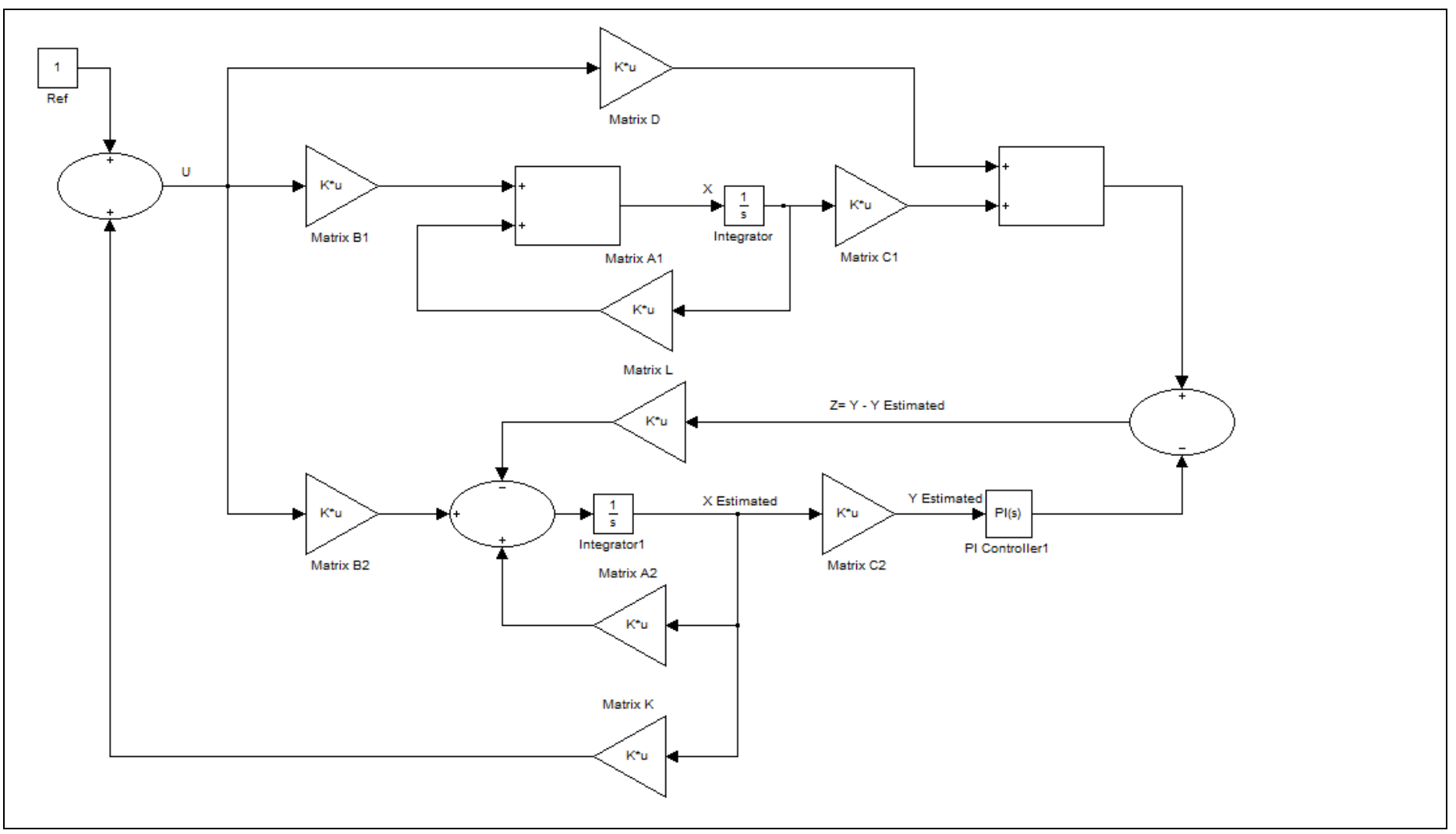

Fig. 3. State estimator function block with PI controller at the error signal of the state estimator

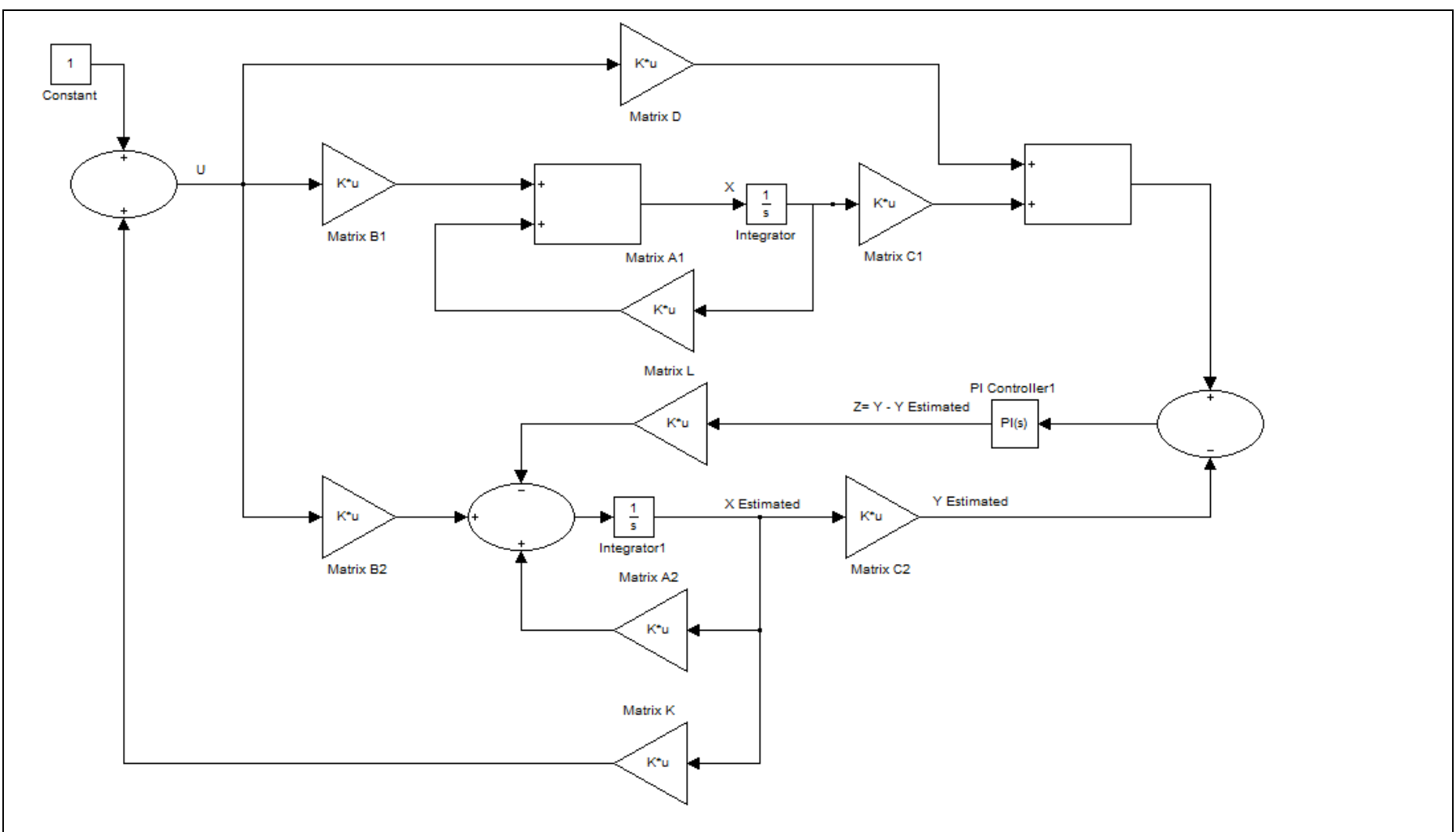

Fig. 4. State estimator function block with PI controller at the output signal of the state estimator 


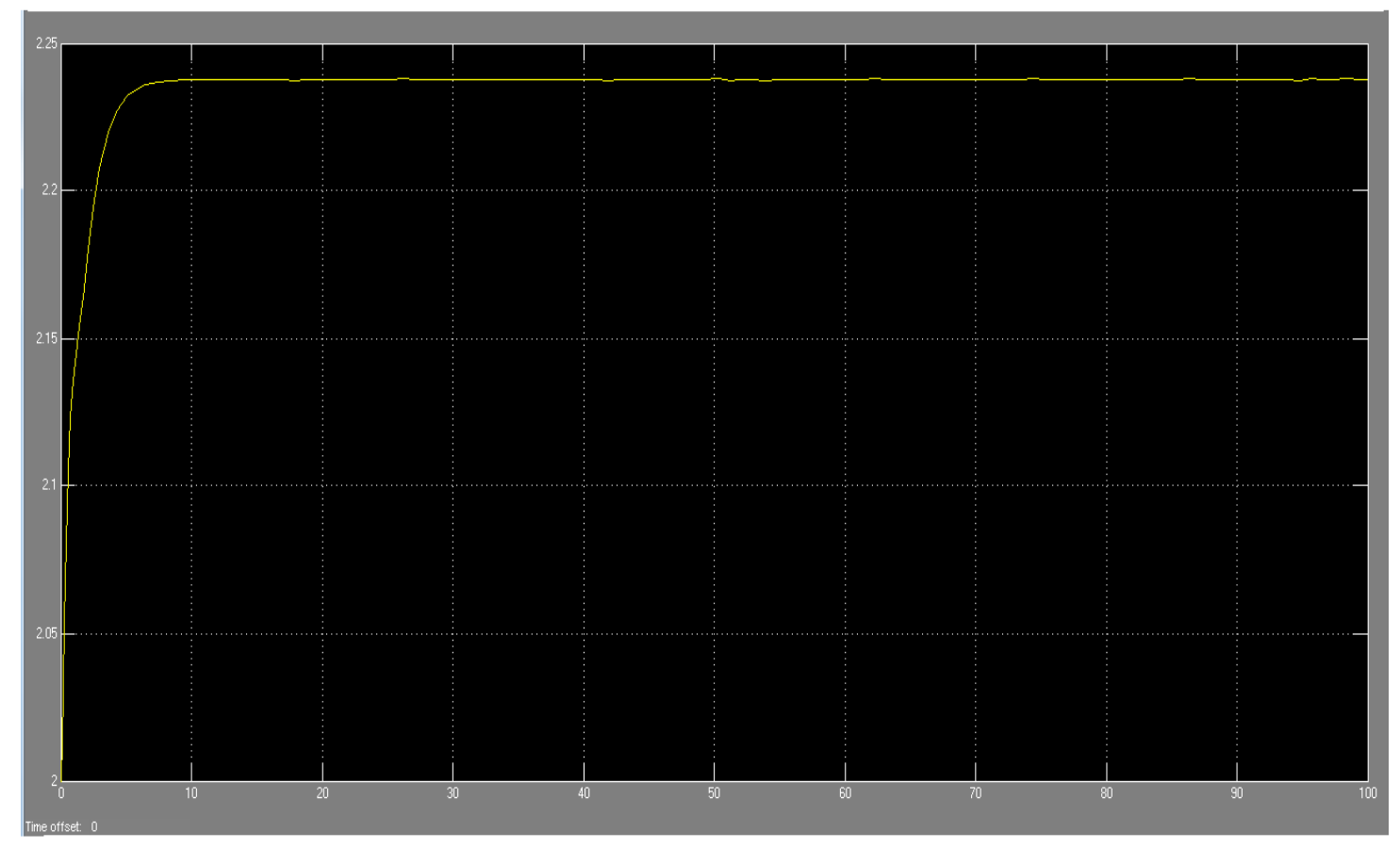

Fig. 5. State Estimator Function Block - Error Signal

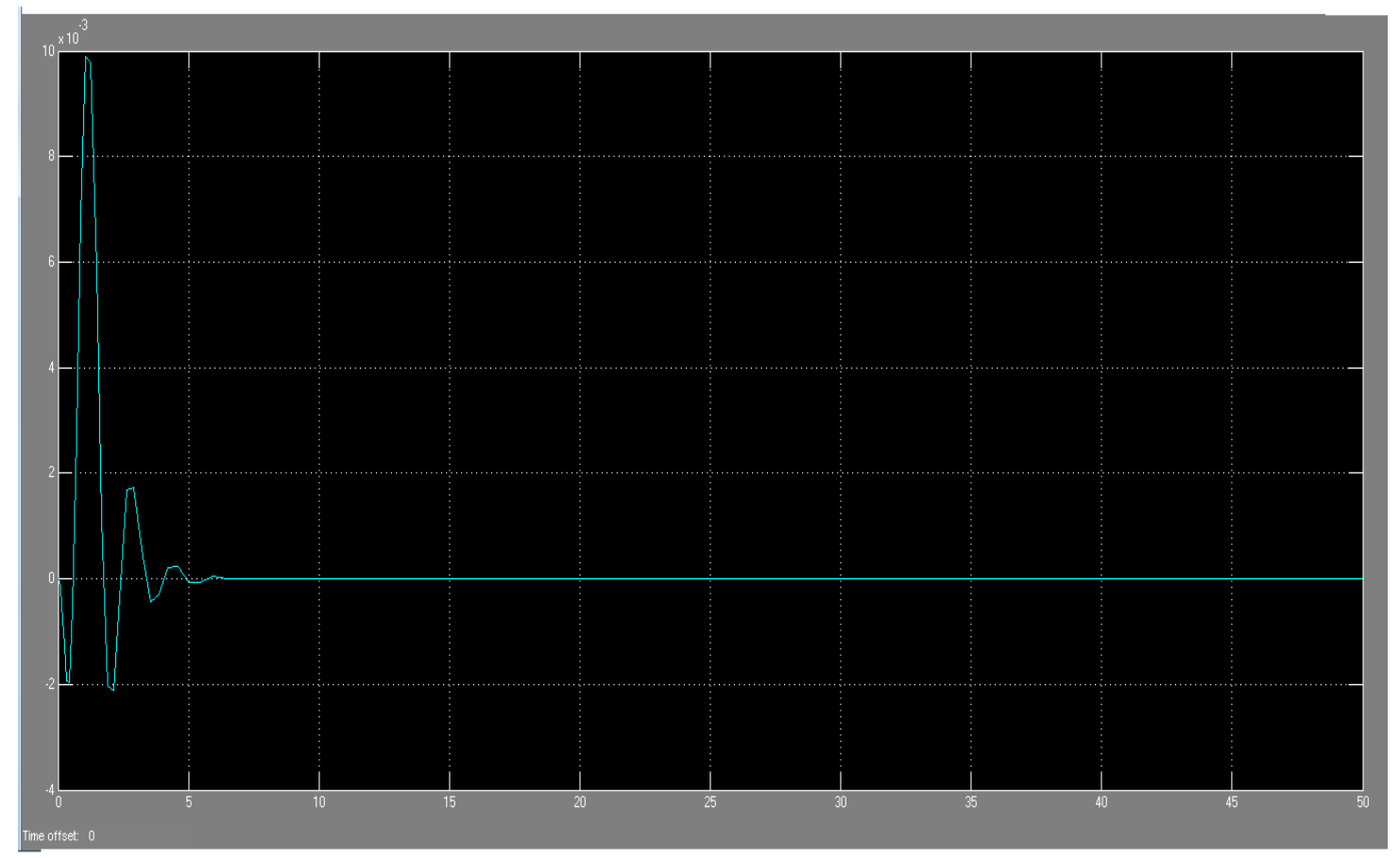

Fig. 6. State estimator function block with PI controller at the input signal of the state estimator - Error signal 


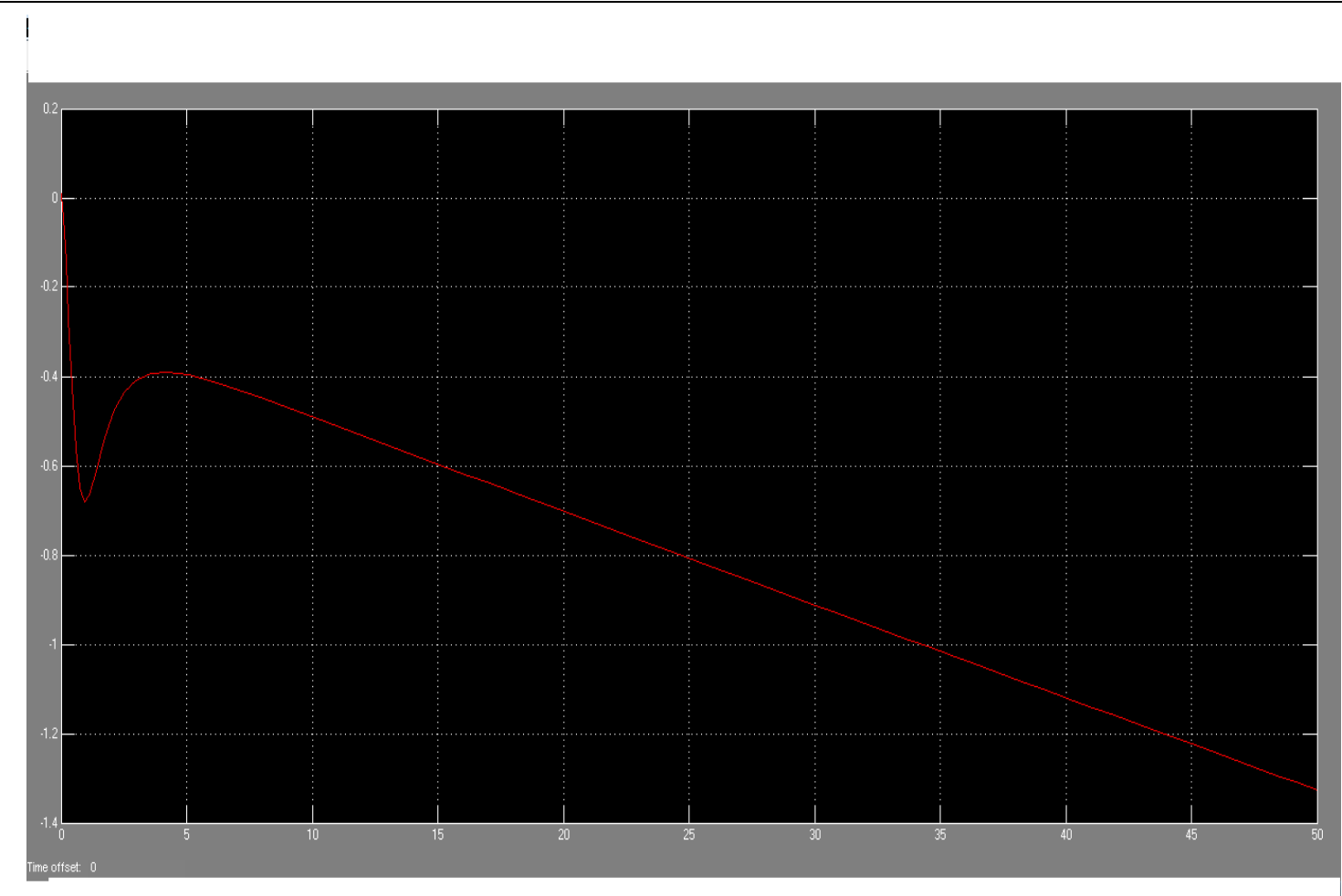

Fig. 7. State estimator function block with PI controller at the error signal of the state estimator-Error Signal

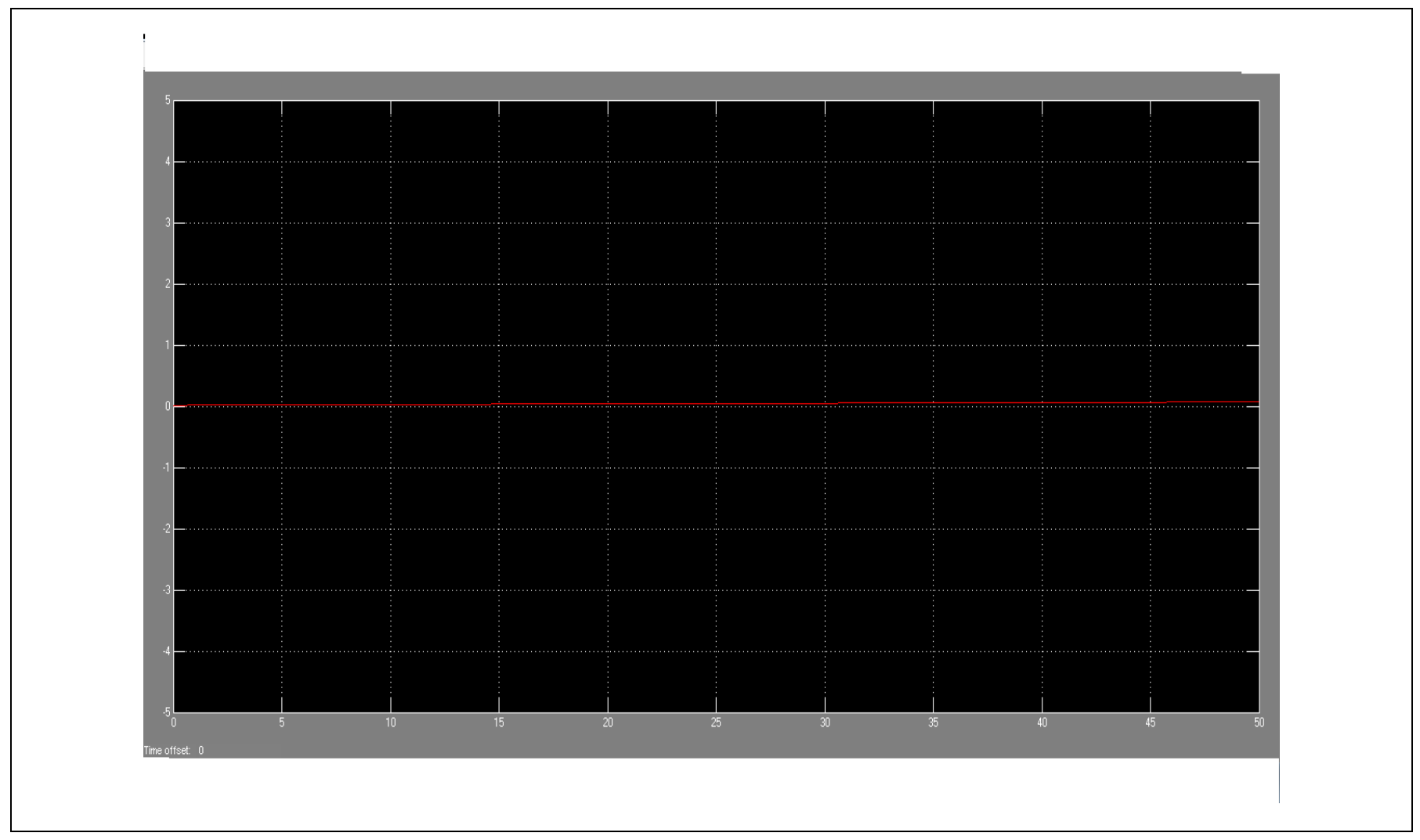

Fig. 8. State estimator function block with PI controller at the output signal of the state estimator - Error signal 